Review

\title{
Plasticity of glucose metabolism in activated immune cells: advantages for melatonin inhibition of COVID-19 disease
}

\author{
Russel J. Reiter ${ }^{*}$, Ramaswamy Sharma1, Qiang Ma1, Changwei Liu², Walter Manucha ${ }^{3}$, \\ Pedro Abreu-Gonzalez ${ }^{4}$, Alberto Dominguez-Rodriguez ${ }^{5}$ \\ ${ }^{1}$ Department of Cell Systems and Anatomy, UT Health Science Center at San Antonio, \\ San Antonio, Texas, USA \\ ${ }^{2}$ Department of Vascular Surgery, Peking Union Medical College Hospital, Beijing, China \\ ${ }^{3}$ Department of Pathology, National University of Cuyo, Mendoza, Argentina \\ ${ }^{4}$ Department of Physiology, Universidad de La Laguna, Tenerife, Spain \\ ${ }^{5}$ Department of Cardiology, Hospital Universitario de Canarias, Tenerife, Spain \\ *Correspondence: reiter@uthscsa.edu, Tel: +1 210-567-3859
}

Running title: Melatonin mechanisms in COVID-19

Received: May 12, 2020; Accepted: June 7, 2020

\begin{abstract}
SARS-CoV-2 has infected hundreds of thousands and killed tens of thousands of people worldwide and it continues to ravage societies as well as fiscal and economic stability of several countries. Currently, several drugs that were designed for other conditions have been repurposed to counter the COVID-19 pandemic. Some have modest efficacy in resisting this disease, but all of them have significant toxicity. Several pharmaceutical companies are rushing to develop vaccines, but their availability is 8 to 12 months in the future. In the meantime, readily available and affordable molecules that will have utility as COVID-19 antidotes are being sought. Recently, several groups independently and almost simultaneously proposed that melatonin should be considered for this purpose and several trials are underway to test whether melatonin is a reliable candidate drug for COVID-19 treatment. In this brief review, we described some potential mechanisms by which melatonin may work to protect against a COVID-19 infection. Of particular note is the likely ability of melatonin to force activated immune cells to abandon aerobic glycolysis in favor of mitochondrial oxidative phosphorylation. Aerobic glycolysis gives proinflammatory activated immune cells, for example, macrophages, an opportunity to produce increased amounts of cytokines which are released as the cytokine storm. The associated significant increase in oxidative stress is accepted as a major contributing factor to the bronchoalveolar dysfunction and pneumonia that occurs in SARS-CoV-2-infected individuals. Melatonin, via similar processes, may reduce the formation of proinflammatory M1 macrophages and convert them to macrophages of the M2 phenotype, which are antiinflammatory. These are not the only mechanisms by which melatonin may protect against the deadly COVID-19 pandemic. Melatonin could also be given as an adjuvant with other toxic pharmaceutical agents with a high likelihood it would reduce their side effects.
\end{abstract}


Key words: glucose metabolism, aerobic glycolysis, oxidative phosphorylation, cytokine storm, macrophage, angiotensin converting enzyme 2 (ACE2).

\section{INTRODUCTION}

Coronaviruses, which includes SARS-CoV-2, are enveloped positive-sense single-strand RNA viruses; their hosts are either mammals or birds. SARS-CoV-2 is responsible for the ongoing pandemic caused by the disease COVID-19. This disease causes mild to severe symptoms which seems to depend in part on the ability of the organism to mount an adequate anti-inflammatory response. The virus depends of trimeric spike proteins which bind to angiotensin converting enzyme 2 (ACE2) in the cell membrane after which it is engulfed by the cell where it creates havoc.

COVID-19 has been and continues to be a major medical challenge to healthcare professionals and scientists. Reliable means to effectively control or combat this deadly infectious virus has not yet been realized. This highly infective virus has distributed itself around the world almost with impunity. The virus is akin to the coronaviruses that are related to other disease outbreaks that have occurred in recent memory, i.e., the SARS (Severe Acute Respiratory Syndrome) and the MERS (Middle East Respiratory Syndrome).

The most common recommendation to slow the spread of COVID-19 is social distancing or quarantine. Such low-tech passive attempts are of value but have a very high adverse effect of devastating the economy of the affected countries and possibly of the world. It is important that more aggressive means be found to prevent or overcome the effects of this virus (and future similar outbreaks) so much of the workforce can stay on the job.

For this purpose, vaccines are urgently being developed but their availability is months to years away; therefore, a vaccine is not an acceptable option for the ongoing pandemic. As an alternate strategy, many currently available conventional drugs are being repurposed to shield against this disease and some have shown qualified success. Likewise, vitamins, and especially high doses of vitamin $\mathrm{C}$ as well as vitamin $\mathrm{D}$, have been suggested as potential combatants for COVID-19 as have other non-prescription agents.

In this special issue, the evidence for the role of melatonin as a potential player in preventing or treating SARS-CoV-2 infection is reviewed. The current report describes some possible mechanisms which may help to justify melatonin's use in prophylaxis or as a treatment for individuals with active COVID-19.

\section{CYTOKINE STORM}

During a coronavirus infection, the inflammatory system becomes excessively zealous in its attempts to quell the infection. This overreaction creates a situation that, rather than being beneficial, produces harm and causes cellular and molecular damage, especially at the level of the lungs but many other organs as well. As a result of alveolar endothelial cell inflammation, surfactant physiology is also disturbed which can cause the alveolar sacs to fill with fluid or collapse. In either case, ventilation dynamics are disrupted leading to depressed oxygen uptake that leads to systematic hypoxemia $(1,2)$. These disturbances contribute to some of the major signs of a SARS-CoV-2 infection such as cough, shortness of breath, and fatigue. 
The specific destructive mediators of a hyperactive inflammatory response are a group of small proteins referred under the umbrella term as cytokines. This group of agents, some of which are highly proinflammatory, include interferons, interleukins, chemokines, tumor necrosis factor, and colony-stimulating factors. Under usual conditions, these proteins participate in intercellular signaling and communication. The specific action depends on the cytokine and the receptor with which it interacts on the target cell. Commonly, individual cytokines have multiple functions. The complex functional networks make it difficult to identify the unique functions of a specific cytokine; they exhibit marked physiological overlap and functional redundancy.

A viral invasion, such as occurs with SARS-CoV-2, induces a dynamic upregulation of the cytokine network which causes extensive molecular and cellular destruction. This overcompensation of the inflammatory response is named the cytokine storm (3). This response often compromises local organ function with the degree of damage and pathophysiology inflicted depending on a number of factors. With the spontaneous or treatment resolution of the infection, normal function can be restored; however, when local inflammation is severe, the organ can develop fibrosis, which causes life-long functional distress.

The sequence of events that occurs during a coronavirus infection of the bronchial tree include viral invasion, activation of dendritic and endothelial cells, and recruitment of other inflammatory cells. These cells synthesize and secrete an abundance of proinflammatory cytokines including interleukins (IL-1 $\beta$, IL-2, IL-6, IL-8), interferons (IFN1 $\alpha$ and IFN-1 $\beta$ ), and tumor necrosis factor- $\alpha$ (TNF- $\alpha$ ) as well as chemokines such as CC motif cytokine 3 (CCL3), CCL6 and interferon gamma-induced protein 10 (IP10). These groups of agents are produced in several cell types including those mentioned above and in macrophages (4, 5). The proinflammatory agents induce excessive amounts of reactive oxygen (ROS) and reactive nitrogen species (RNS) leading to extensive nitro-oxidative stress which contributes significantly to the respiratory pathophysiology.

\section{MITOCHONDRIA AS A SOURCE OF INTRACELLULAR MELATONIN}

Melatonin has long been known as a synthetic and secretory product of the vertebrate pineal gland where it is uniquely produced and released at night (6). This day:night variation leads to a marked circadian rhythm of melatonin in the cerebrospinal fluid (CSF) and in the blood with the duration of the elevated nighttime rise being proportional to the duration of darkness $(7,8)$.

Experimental evidence indicates, however, that melatonin synthesis is not restricted exclusively to the pineal gland. Within slightly more than a decade after its identification in bovine pineal tissue, melatonin was also discovered to be present in the vertebrate retina and soon thereafter in many other organs and cells (9) and in non-vertebrate species (10). In 1995, melatonin was immunocytochemically identified in a bacterium, Rhodospirillum rubrum (11). This discovery was of special importance since mitochondria of present-day animals and plants theoretically evolved from prokaryotic bacteria after their ingestion by early eukaryotes; this is known as the endosymbiotic theory for the origin of mitochondria and is widely accepted by the scientific community (12). Since bacteria contain and presumably synthesize melatonin, it was subsequently speculated that mitochondria of all cells have retained the synthetic machinery for melatonin production (13). This theory draws additional support from the observation of exceptionally high levels of melatonin in mitochondria of mammalian cells relative to the much lower concentrations in other subcellular compartments (14). 
Direct evidence subsequently verified mitochondria as a site of melatonin synthesis. Using isolated oocyte mitochondria, He et al. (15) showed that they synthesized melatonin only when they were incubated in a medium containing serotonin (5-HT), a required precursor of melatonin. Also, Suofu and coworkers (16) reported that the proteins for the two enzymes required for the conversion of 5-HT to melatonin, i.e., arylalkylamine $\mathrm{N}$-acetyltransferase (AANAT) and acetylserotonin methyltransferase (ASMT), are located in mammalian brain mitochondria. The findings of $\mathrm{He}$ and colleagues (15) are especially revealing since they used isolated oocyte mitochondria in which to examine melatonin production. Given that mitochondria in all mammalian cells are of maternal (oocyte) origin it is reasonable to assume that the synthesis of this critical molecule was retained in all mitochondria-containing cells.

As currently envisaged, vertebrate cells have two pools of melatonin (17). The pineal gland produces and secretes melatonin into the CSF and blood, the amount of which varies according to the light:dark cycle. The resulting rhythm impacts circadian and circannual functions in vertebrates (Figure 1). The second pool of melatonin is derived from the mitochondria of all cells. In these organelles, melatonin is not synthesized in a circadian manner $(14,16)$ and it does not enter the CSF or blood. Rather, this melatonin is used locally as an antioxidant, a genomic regulator, etc. Melatonin from these cells may leak into the extracellular environment where it presumably has autocrine and paracrine actions $(18,19)$ (Figure 1). Herein, we hypothesize that mitochondria-produced melatonin plays a critical role in squelching the cytokine storm.

Proinflammatory cytokines activate immune cells such as macrophages and dendritic cells which alter their metabolism to accommodate the production of the massive amounts of cytokines required to mediate the cytokine storm. Hence, these cells switch from using mitochondrial oxidative phosphorylation (OXPHOS) for ATP production to cytosolic aerobic glycolysis. This metabolic reprogramming is similar to that observed in solid tumor cells, where it is referred to as the Warburg effect. Interrupting mitochondrial OXPHOS allows the immune cells to rapidly, albeit, less efficiently, generate the necessary energy (ATP) to support the elevated synthetic activity of these cells. In addition to supporting the rapid production of ATP, it activates the pentose phosphate pathway which improves the accelerated protein synthesis, including cytokines (20) (Figure 2).

Under non-inflammatory conditions, the macrophages and dendritic cells take up glucose after which in a multistep process it is metabolized to pyruvate. The bulk of the pyruvate then is transported into the mitochondria where it is converted to acetyl-coenzyme A (acetyl-CoA) by the enzyme pyruvate dehydrogenase complex (PDC). Acetyl-CoA enters the tricarboxylic acid cycle (TCA) and eventually supports OXPHOS. However, acetyl-CoA also controls mitochondrial melatonin production.

Mitochondria not only produce melatonin locally, but when there are elevated circulating levels in the blood or in the CSF (or in the incubation fluid of in vitro studies), it readily enters cells and gains access to the mitochondria. The first indirect evidence for its entrance into mitochondria were the findings that melatonin added to cultured cells quickly altered mitochondrial respiration (21) and suppressed mitochondrial ROS concentrations and the associated oxidative stress (22). How melatonin enters cells may relate to its high lipid solubility allowing it to merely diffuse through bilipid membranes. Other options have also been proposed. Evidence garnered by Hevia et al. (23) supports the transport of melatonin into cells by a glucose transporter (GLUT1). An alternative route by which melatonin enters cells and mitochondria is via the oligopeptide transporters, PEPT1 and PEPT2 (24). This detailed study strongly supports these transporters as being relevant to the intracellular concentrations of melatonin, particularly 
at the level of the mitochondria since in this case melatonin uptake is achieved against a concentration gradient. This is consistent with the high levels of mitochondrial melatonin as reported by Venegas et al. (14) and more recently by Acuna-Castroviejo and colleagues (25). While the findings of Huo et al. (24) are of great interest, they used exclusively cancer cells (five types) to document melatonin transport to the intracellular environment. It is presumed that the PEPT1 and PEPT2 also exist in healthy cells and function similar to those in cancer cells. If melatonin is avidly taken up by mitochondria of all cells, it can be classified as a mitochondriatarget molecule (26); this would make melatonin a formidable force in protecting these critical organelles from oxidative damage $(18,27,28)$.

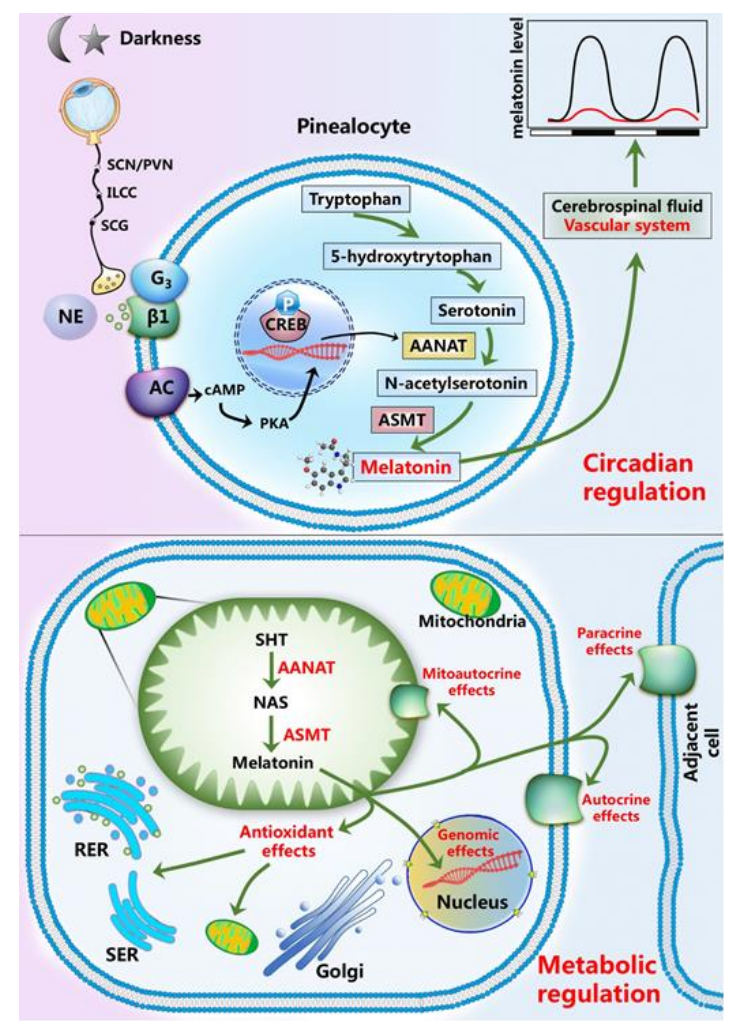

Fig. 1 Two pools of melatonin, a releasable pool from the pineal gland, and a nonreleasable pool from mitochondria of perhaps all other cells.

Pineal melatonin synthesis is rhythmic and reliant on the prevailing light:dark cycle as perceived by specialized retinal photoreceptors, the intrinsically photoreceptive ganglion cells. The neural connection between the eyes and the pineal gland involve the suprachiasmatic nuclei (SCN, the master circadian clock) and the central and peripheral sympathetic nervous system. Melatonin, which is produced especially during darkness in the pineal gland, is quickly discharged into the CSF and blood with the rise in CSF level greatly exceeding that in the blood. The melatonin rhythm provides an important circadian signal to peripheral oscillators which exist in every cell. The non-releasable (not released into the blood or CSF) pool is derived from the mitochondria of presumably every cell. In mitochondria, melatonin synthesis is nonrhythmic but may be inducible. Mitochondria-derived melatonin has local intracellular actions at the mitochondrial level (mitoautocrine effects) and genomic actions. It also functions intracellularly as an antioxidant. Melatonin from these cells may be discharged into the local cellular microenvironment where it may have autocrine and paracrine actions. 


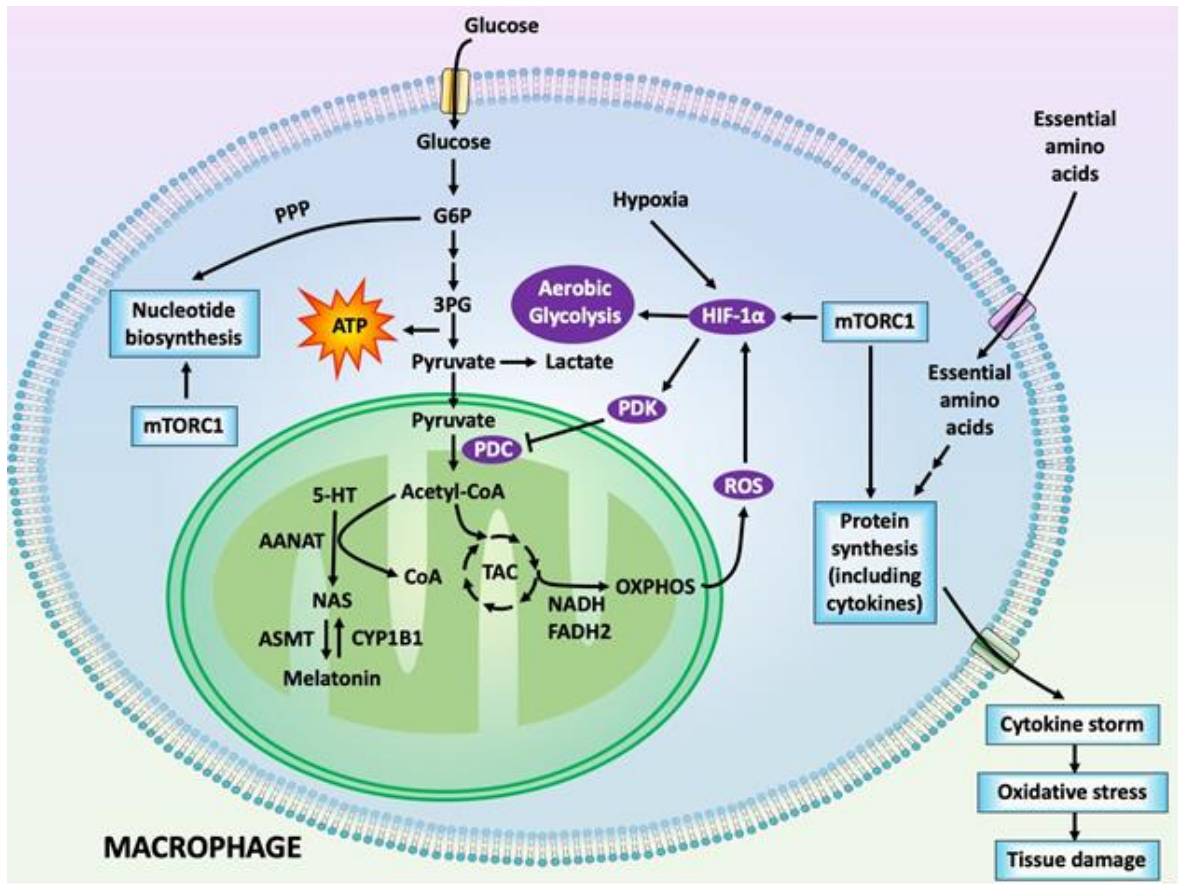

Fig. 2 Metabolism of macrophages (both M1 and M2 are represented in this figure).

The proinflammatory M1 macrophage utilizes aerobic glycolysis for rapid ATP production and active biomolecule generation such as ATP and proteins including cytokines; they then release massive amounts of cytokines (cytokine storm) which cause tissue damage via oxidative stress and other means. Because they use aerobic glycolysis in lieu of oxidative phosphorylation, M1 macrophages also do not synthesize melatonin in their mitochondria while M2 macrophages do generate melatonin at this level. M1 macrophages fail to produce melatonin since they are incapable of synthesizing acetyl-CoA in their mitochondria because the enzyme complex that converts pyruvate to acetyl-CoA, pyruvate dehydrogenase complex (PDC) is strongly downregulated due to activation of pyruvate dehydrogenase kinase (PDK) (note red $X)$. When acetyl-CoA cannot be produced in the mitochondria of M1 macrophages, melatonin failure occurs since acetyl-CoA is a required co-factor/substrate for the rate limiting enzyme for melatonin synthesis, arylalkylamine $N$-acetyltransferase (AANAT). Since both melatonin and $N$-acetylserotonin (NAS) are potent anti-inflammatory agents and ubiquitous antioxidants, their loss exaggerates the cytokine storm (it produces the "perfect" cytokine storm) because it is unopposed by melatonin and NAS. The resulting massive damage that occurs in the lungs of individuals infected with SARS-CoV-2 is generally considered a result of the ("perfect") cytokine storm. Exogenously-administered melatonin inhibits PDK and restores the intercellular cytokine and oxidant balance allowing for a reduction in bronchoalveolar damage. When PDC is disinhibited, as occurs in M2 macrophages, mitochondrial acetyl-CoA is produced, melatonin is synthesized and oxidative phosphorylation (OXPHOS) produces the bulk of the ATP with a limited generation of toxic cytokines. ASMT = acetylserotonin methyltransferase; EGF = epithelial growth factor; GMCSF = granulocyte macrophage colony stimulating factor; IL = interleukin; INF = interferon; LPS = lipopolysaccharide; $P D G F=$ platelet-derived growth factor; $R N S=$ reactive nitrogen species; $R O S=$ reactive oxygen species; $T G F=$ transforming growth factor. 


\section{MELATONIN ALTERS IMMUNE CELL METABOLISM}

The first evidence that melatonin impacted aerobic glycolysis was published in 2014 by Blask (29) and colleagues. They showed that the abundant glucose uptake and its conversion to lactate (an indicator of the Warburg effect) by solid breast tumors exhibited a marked circadian rhythm. While it was obvious that during the day these human tumors, growing in immune-compromised rats, clearly used Warburg-type metabolism, at night during darkness they did not, i.e., they had abandoned aerobic glycolysis in favor of mitochondrial oxidative phosphorylation to generate ATP. This novel day:night difference had not been previously observed in any tissue, perhaps because it had never been suspected and, therefore, never examined $(30,31)$. Equally important, Blask et al. (29) found that the conversion of daytime aerobic glycolysis to nighttime OXPHOS was totally dependent on the nocturnal rise in circulating melatonin; when the elevation of melatonin in the blood was suppressed by exposing the animals to light at night, tumor aerobic glycolysis proceeded 24 hours per day; thus, the tumors never utilized mitochondrial OXPHOS. Since under these conditions, the tumors employ aerobic glycolysis continuously throughout the 24-hour period, they grew much faster since this type of metabolism rapidly produces the necessary energy (ATP) for rapid cell proliferation due to accelerated nucleotide and protein synthesis (32). Since the report by Blask et al. (29), other authors have provided credible experimental evidence that melatonin reverses aerobic glycolysis in cancer cells $(33,34)$.

The importance of these observations came into clearer focus when melatonin was found to be synthesized in mitochondria $(15,16)$; this likely occurs in every cell $(13,35)$. In normal cells, pyruvate, a product of glucose metabolism, enters the mitochondria where it is metabolized to acetyl-coenzyme A (acetyl-CoA). Acetyl-CoA is a critical molecule in mitochondria because, as noted above, it feeds into the tricarboxylic acid cycle (TCA) which, in turn, supports OXPHOS and ATP synthesis. Additionally, acetyl-CoA is required for intra-mitochondrial melatonin production since it is a necessary co-factor/substrate for the rate limiting enzyme, AANAT for the conversion of 5-HT to N-acetylserotonin (NAS), the precursor of melatonin (36) (Figure 2). With the suppression of AANAT activity the cells are deprived of both NAS and melatonin. Since both these molecules are efficient radical scavengers and indirect antioxidants, the mitochondria and, therefore, the cells are vulnerable because their defense against the toxic cytokines and free radicals is greatly diminished (27, 28, 37-39).

In some pathological states, e.g., in cancer cells and in cells infected with SARS-CoV-2 virus, cellular glucose metabolism is hijacked such that pyruvate is prevented from being converted to acetyl-CoA $(30,31)$ and, as mentioned, local NAS and melatonin production is severely depressed making the cells capable of increasing their production of cytokines and ROS (39). In the case of M1 macrophages in the lungs of COVID-19 infected patients, this leads to excessive bronchoalveolar damage and the associated deadly pneumonia which these people develop.

The ability of exogenously-administered melatonin to protect against a COVID-19 infection likely stems, at least in part, from its ability to reverse aerobic glycolysis in the infected cells allowing them to adopt mitochondrial OXPHOS and to produce intra-mitochondrial melatonin (19). The locally-produced melatonin, coupled with supplemental melatonin, aids in resisting the molecular and cellular damage caused by the cytokine storm and thereby improving the outcome of patients affected by COVID-19. Since melatonin can also be reversed metabolized to its precursor, NAS, both molecules may have protective roles against COVID-19 (39).

In this scheme, we propose that activated M1 macrophages are those that display aerobic glycolysis and release the cytokine storm. Supplemental melatonin inhibits aerobic glycolysis in 
proinflammatory M1 macrophages and converts them to anti-inflammatory M2 macrophage phenotype (Figure 3).

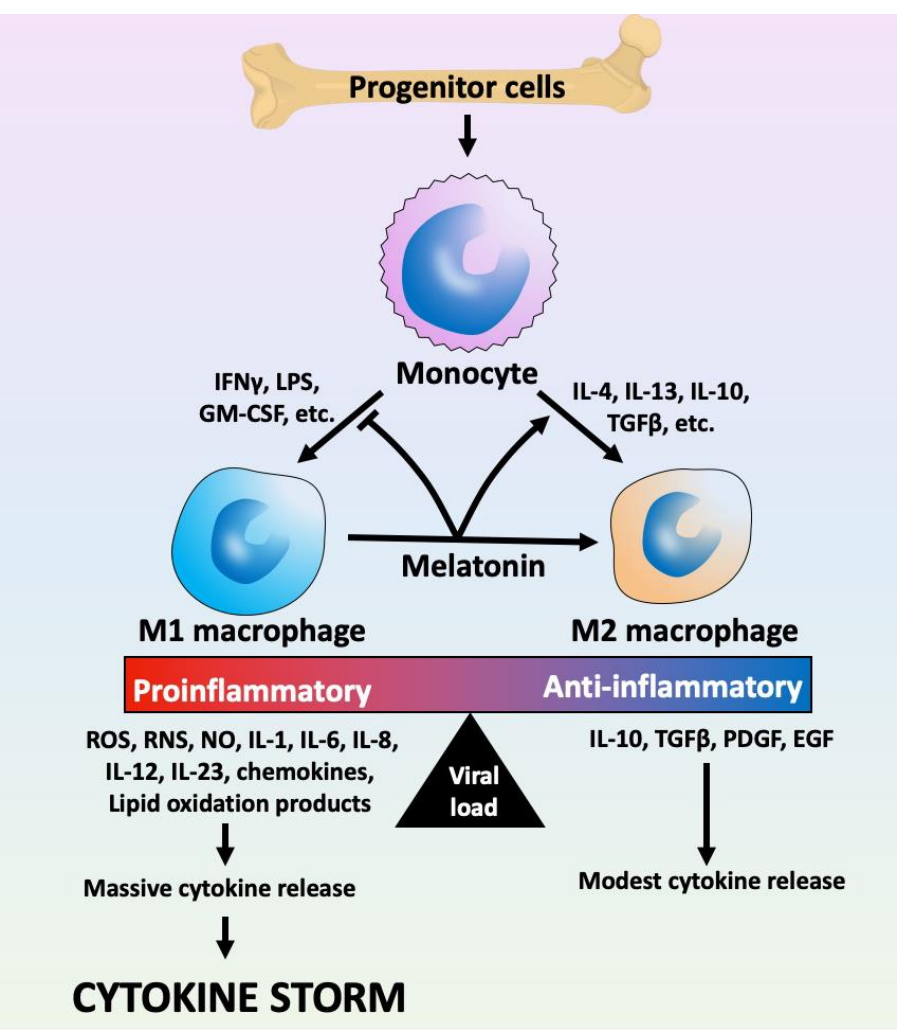

Fig. 3 Development of the cytokine storm in macrophages.

Blood monocytes derive from undifferentiated hematopoietic tissue in the bone marrow. During a SARS-CoV-2 infection, monocytes are transformed into activated macrophages (MI macrophages) which are highly proinflammatory. The M1 macrophages utilize aerobic glycolysis for rapid ATP production and abundant cytokine/chemokine synthesis which are released and contribute in a major way to the cytokine storm which damages the bronchoalveolar tissue because of oxidative stress. In addition to M1 macrophages, activated lymphocytes, endothelial cells and fibroblasts contribute to the cytokine storm by also producing large amounts of cytokines/chemokines. Monocytes can also differentiate into less active macrophages (M2 macrophages). These cells utilize mitochondrial oxidative phosphorylation for ATP production; they also produce cytokines but in such smaller amounts than MI macrophages. Exogenously-administered melatonin presumably converts $M 1$ to M2 macrophages thereby reducing the cytokine storm and the associated lung injury in COVID-19 disease. Moreover, because it is an efficient direct ROS/RNS scavenger, melatonin (as well as its precursor, $\mathrm{N}$-acetylserotonin) also protects the lungs from damage via this action. The significance of aerobic glycolysis typical of M1 macrophages versus oxidative phosphorylation by M2 macrophages is important in COVID-19 disease and melatonin hypothetically shifts M1 to the anti-inflammatory $M 2$ phenotype which does not promote an inflammatory storm. EGF = epithelial growth factor; GMCSF = granulocyte-macrophage colony stimulating factor; IL = interleukin; $L P S=$ lipopolysaccharide; $N O \bullet=$ nitric oxide; $P D G F=$ platelet-derived growth factor; $R N S=$ reactive nitrogen species; $R O S=$ reactive oxygen species; $T N F=$ tumor necrosis factor; $T G F=$ transforming growth factor. 
In the lungs of COVID-19 patients, it is presumed that the bulk of the macrophages are of the M1 phenotype and exogenously-administered melatonin takes advantage of their metabolic plasticity and reverts them back to the M2 phenotype making this indoleamine effective as a treatment for COVID-19 as also seems to be the case in solid tumors (31). However, other drugs, which may impact the replication or uptake of the SARS-CoV-2 virus into cells, may also be effective in influencing the progression of COVID-19 disease. When these drugs are used, however, it would seem judicious to combine them with melatonin which works via a different means and would also protect the cells from the toxicity of the repurposed prescription medications, resulting in synergism.

Some vitamins have been proposed as being potentially useful agents to protect against SARS-CoV-2 infection. In particular, high dose vitamin $\mathrm{C}$, because of its antioxidant potential, is reported to benefit COVID-19 patients (40). Also, vitamin D has generated interest as a potential treatment for COVID-19 (41, 42). Indeed, melatonin and vitamin D may have some similar actions since both molecules bind to a common nuclear receptor (43).

\section{MELATONIN AND MACROPHAGE POLARIZATION}

A major cause of death in SARS-CoV-2 infected individuals is the intense inflammatory response in the lungs which leads to progressive and severe damage referred to as acute respiratory distress syndrome (ARDS) (44). The pathophysiology of the compromised respiratory function in these patients is believed to be related to the cytokine storm as described above $(4,45)$. Macrophage activation, which results in the formation of the M1 phenotype, is a substantial contributor to the exaggerated inflammatory response and, as a result, to deadly pneumonia in COVID-19 patients (45).

An association between melatonin and macrophages is well established (68). The hematopoietic precursors of the monocyte/macrophage lineage synthesize melatonin as do mature macrophages $(4,46)$, including macrophages associated with the lungs (47-49). Monocytes also differentiate into dendritic cells (50). Dendritic cells, like activated macrophages, may be highly proinflammatory but the role of melatonin in modulating dendritic cell cytokine production is ill-defined.

Macrophages have a high degree of plasticity such that they can readily switch between two markedly different phenotypes with opposed actions, i.e., proinflammatory (M1) and antiinflammatory macrophages (M2) depending on the microenvironment in which the cell is located (51) (Figure 3). When activated, which may be due to exposure to lipopolysaccharide (LPS), interferon (IFN) $-\gamma$, or other agents, they are polarized to the M1 phenotype and produce high levels of proinflammatory cytokines such as IL-1 $\beta$, IL6, TNF- $\alpha$ and others (52). M1 microphages also upregulate inducible nitric oxide synthase (iNOS) resulting in the generation of nitric oxide (NO•), a gaseous free radical (53). Also, activated macrophages stimulate NADPH oxidase as well as myeloperoxidase to generate the partially-reduced oxygen derivatives, the superoxide anion $\left(\mathrm{O}_{2}{ }^{--}\right)$and hypochlorous acid (Figure 4). $\mathrm{O}_{2}{ }^{--}$can be reduced to hydrogen peroxide $\left(\mathrm{H}_{2} \mathrm{O}_{2}\right)$ which via the Haber-Weiss reaction leads to the formation of the highly destructive agent, the hydroxyl radical $(\bullet \mathrm{OH})$. Also, of importance is that $\mathrm{NO} \bullet$ couples with $\mathrm{O}_{2}{ }^{\bullet-}$ to produce the powerful oxidizing agent, the peroxynitrite anion $\left(\mathrm{ONOO}^{-}\right)$. Both the $\bullet \mathrm{OH}$ and $\mathrm{ONOO}^{-}$are highly effective at inducing oxidative stress. Thus, M1 macrophages are proinflammatory while also being pro-oxidative. 


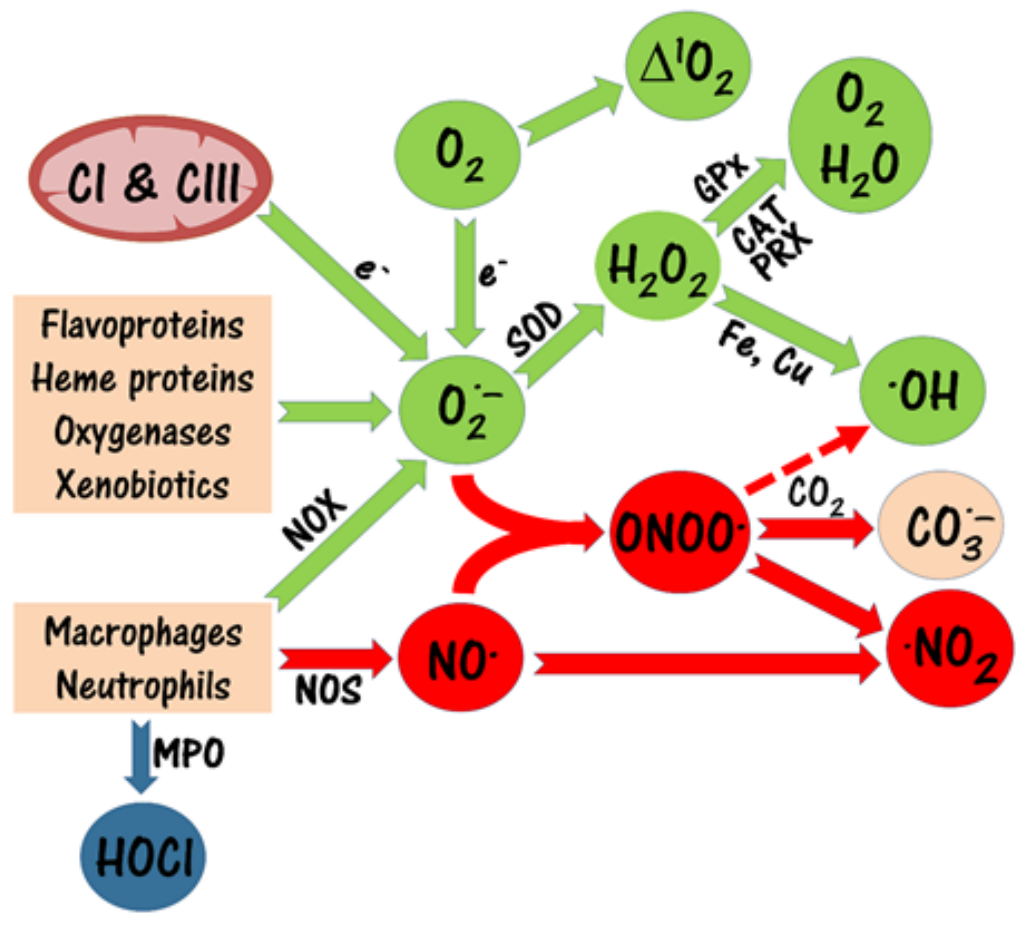

Fig. 4 Damaging reactive species that are formed during a cytokine storm.

These toxic agents account for much of the damage that occurs in the lungs, and other tissues, infected with SARS-CoV-2. The generated reactants are either reactive oxygen-based (reactive oxygen species or ROS) (highlighted in green) or reactive nitrogen-based (reactive nitrogen species or RNS) (highlighted in red). These agents are derived from multiple sources including CI and CIII (complexes I and III) of the respiratory chain, during protein metabolism, by oxidases and during xenobiotic metabolism. Activated macrophages and other blood cell elements generate large amounts of ROS/RNS during a cytokine storm. Melatonin, a multifunctional antioxidant, directly scavenges ROS/RNS or indirectly metabolizes them to harmless products and reduces nitro-oxidative stress. $C A T=$ catalase; $G P x=$ glutathione peroxidase; NOS and NOX = nitric oxide synthetase; $P R X=$ peroxiredoxin; $S O D=$ superoxide dismutase. Solid arrows indicate confirmed reaction; hyphenated arrow indicates likely reaction.

Melatonin is a well-known downregulator of iNOS, suppressor of signaling events that enhance inflammation (54), and a widely-confirmed free radical scavenger $(27,55,56)$ in addition to promoting antioxidant enzymes, particularly mitochondrial superoxide dismutase (mSOD) (57). M1 phagocytes avoid acetyl-CoA and ATP production in the mitochondria because they switch to aerobic glycolysis (57). As a consequence of that switch, the mitochondria are incapable of producing melatonin $(19,31)$. In the absence of this critical antiinflammatory and antioxidant at the intracellular level, the cytokine storm can proceed mainly without resistance in the lungs and in other organs thereby contributing to a bleak situation of extensive and progressive molecular damage $(3,58)$ and organ deterioration.

Melatonin blocks the polarization of macrophages to the proinflammatory M1 phenotype via several means that have been at least partially defined $(59,60)$. Activation of the toll-like receptor 4 (TLR4), as occurs when cells are exposed to LPS, leads to enhanced stimulation of intracellular NF- $\mathrm{KB}$ signaling; this involves the myeloid differentiation primary response gene 
(MyD88)-signaling pathway which strongly influences proinflammatory cytokine synthesis (61). By inhibiting the phosphorylation of inhibitory $\kappa \mathrm{B}(\mathrm{I} \kappa \mathrm{B})$, melatonin interferes with the release of $\mathrm{NF}-\kappa \mathrm{B}$ and its translocation into the nucleus $(62,63)$ where it would typically target $T n f-\alpha$ and I6- $1 \beta$ gene expression among others. Additionally, melatonin converts the proinflammatory M1 macrophage phenotype to the anti-inflammatory M2 macrophage via activation of the STAT-6 signaling pathway (64). Although mechanistically only poorly defined, melatonin also modulates macrophage polarization via processes that involve miRNAs, in particular, miR-155 and miR-146, with the possibility being left open that other miRNAs may also be involved with M1 to M2 conversion $(65,66)$.

There is limited specific information regarding the actions of melatonin in the polarization of macrophages. In the event of an excessive inflammatory response, such as occurs during the cytokine storm is characteristic of a SARS-CoV-2 viral infection. Melatonin may have a critical anti-inflammatory role by controlling macrophage polarization. The specific molecular events by which melatonin controls the balance between proinflammatory M1 macrophages and antiinflammatory M2 macrophages are yet to be defined. The reader who wants additional information can consult the following recent publications $(64,67-69)$ and see figure 3.

\section{MELATONIN AND ANGIOTENSIN CONVERTING ENZYME}

Angiotensin-converting enzyme 2 (ACE2) reduces cellular pathologies resulting from intensive and prolonged inflammation by suppressing TNF- $\alpha$ and IL-6 (70). Cellular damage occurs when ACE2 is downregulated which causes the overactivation of the angiotensin II (ANG) II/AT1R (angiotensin type 1 receptor) axis with the destructive effects of ANGII contributing to multiorgan dysfunction initiated by what is referred to as the macrophage activation syndrome (MAS; production of M1 macrophages) which culminates in the cytokine storm (71). The SARS-CoV-2 virus not only prevents ACE2 from entering into cells but also downregulates ACE2 (72) which contributes to ARDS. ACE2 is widely distributed including in the lungs where it has been identified in a variety of cells including vascular endothelium and the associated smooth muscle cells of these vessels and in airway epithelium (73). ACE2 protects the lungs from damage following the exposure of rodents to sepsis-generating molecules (74). Since SARS-CoV-2 virus dysregulates ACE2, this change may be responsible for the lung damage and pneumonia following COVID-19 disease development. Similarly, any molecule that overcomes ACE2 suppression may reduce the severity of lung infections in COVID-19.

Initially, Zhang et al. $(75)$ and then others $(56,76,78)$ predicted that melatonin may be an effective treatment for COVID-19; it could do so by preserving ACE2 expression not only in the lungs but in other tissues where it is located, e.g., heart and kidneys. The literature related to the topic of melatonin's action on ACE2 is meager with a single report claiming maternal melatonin treatment during pregnancy enhances ACE2 levels in 6-week-old offspring (79). Despite this very incomplete evidence, the possibility of a direct association between melatonin and bronchial cell ACE2 activity should be borne in mind especially since viral-mediated lung damage is mediated by inhibition of ACE2 in other models of viral infections $(80,81)$.

Rho GTPase and its downstream signaling molecule ROCK (Rho-kinase) are involved in many cellular functions especially when RhoA/ROCK signaling is activated. Based on current evidence, Rho-kinase is upregulated in the inflamed lung and, as a result, inhibition of this enzyme may be beneficial as a treatment for acute lung injury (ALI) and ARDS (82). Under other experimental conditions, melatonin is known to downregulate Rho-associated protein 
kinase expression (83-85). This action of melatonin should be included as a potential mechanism by which it impedes SARS-CoV-2 infection.

\section{CONCLUSIONS AND PERSPECTIVES}

Considering the large number of individuals who have been infected with SARS-CoV-2 virus, the resulting pandemic, which has also ruined the economic base of many countries, must be considered one of the worst medical tragedies on record. COVID-19 not only compromises respiratory physiology, which is of current greatest concern, but also damages many other organs as well, e.g., heart, kidneys, liver, central nervous system, etc.

Medical professionals are working feverishly to identify already-existing medications to combat this disease and especially, to prevent death. Although a number of drugs have been repurposed or used as COVID-19 treatments, none have proven highly or reliably effective in curtailing the SARS-CoV-2 infection. The development of vaccines has also been "fast-tracked" so they can become available in the shortest possible time. But at best, this could be 8 to 12 months or longer in the future. This time delay is not helpful for the treatment of the current pandemic. Obviously, a beneficial treatment for COVID-19 is needed today.

Melatonin, an endogenously produced molecule, has been shown to defer other virus-related diseases in experimental animals and plants $(86,87)$. Indeed, according to the knowledge of the authors, no virus has been identified where melatonin has not been proven effective in quelling its effects. Moreover, the molecular damage resulting from COVID-19 disease relates to its marked stimulation of inflammation (the cytokine storm) which also leads to the extensive nitrooxidative stress that results. Given that melatonin is well established as an anti-inflammatory and antioxidant molecule along with its anti-viral (but not viricidal) actions, herein we proposed, as others have also done, the use of melatonin either alone or in combination with other drugs, to improve the outcome of individuals afflicted with SARS-CoV-2 and provide information on melatonin's potential actions which will help to justify its use as a potential COVID-19 treatment.

In addition to its proven mechanisms of action, melatonin has other advantages for use as a COVID-19 treatment: a) melatonin has a very high safety profile even when given in massive amounts, so overdosing is not an issue; b) melatonin can be administered via multiple noninvasive routes; c) the shelf life of melatonin is very long and its refrigeration is not required; $d$ ) it is readily available in pharmacologically-available purity; e) melatonin can be selfadministered, a major advantage in remote areas or for individuals who are home-bound; f) melatonin may not only protect against acute lung injury but also the long-term respiratory damage associated with a COVID-19 infection, e.g., fibrosis; g) melatonin protects the lungs as well as all other organs, the damage of which contributes to multiple organ failure; and h) if given in combination with repurposed prescription medications, melatonin may reduce the side effects of those drugs.

The COVID-19 pandemic has caused a worldwide crisis. Healthcare professions must be innovative at times like these especially considering the current dire circumstances. While melatonin is not yet guaranteed as an effective treatment, it likely would be useful and is unlikely to do any harm.

The ability of melatonin to suppress the hyperinflammatory response referred to as the cytokine storm is consistent with reports on the current death rate of infected individuals. In general, elderly humans have the highest mortality incidence of those infected with the virus. 
The levels of endogenously-produced melatonin wanes with increasing age, at least in the pineal gland and perhaps in the mitochondria of other cells as well. This being the case, older patients may be more seriously affected by a COVID-19 infection which increases both the morbidity and mortality of this population. A just published report describes a therapeutic algorithm for the treatment of COVID-19 with melatonin (78) while another publication suggested the use of melatonin in combination with prescription drugs $(75,88)$.

Although probably less concerning than the advanced age issue, even young individuals exposed to light at night, such as nightshift workers (who are frequently the health caregivers), have reduced endogenous melatonin production judging from the depressed circulating melatonin levels. Potentially these individuals may exhibit a more serious infection if they contract the SARS-CoV-2 virus.

\section{ACKNOWLEDGEMENTS}

None

\section{AUTHORHSIP}

RJR and ADR conceived the idea and RJR wrote the first draft which was read by all coauthors who offered their suggestions on the interpretation of data and the inclusion of references. All authors read and approved the final version of the manuscript. RJR designed the figures and they were put into final form by QM.

\section{CONFLICT OF INTEREST}

The authors declare no conflicts of interest.

\section{REFERENCES}

1. Luks AM, Freer L, Grissom CK, et al. (2020) COVID-19 lung injury is not high altitude pulmonary edema. High Alt. Med. Biol. in press.

2. Thompson BT, Chambers RC, Liu KD. (2017) Acute respiratory distress syndrome. N. Engl. J. Med. 377: 1904-1905.

3. Tisoncik JR, Korth MJ, Simmons CP, et al. (2012) Into the eye of the cytokine storm. Microbiol. Mol. Biol. Rev. 76: 16-32.

4. Cheung CY, Poon LLM, Ng IHY, et al. (2005) Cytokine responses in severe acute respiratory syndrome coronavirus-infected macrophages in vitro: possible relevance to pathogenesis. J. Virol. 79: 7819-7826.

5. Chu H, Zhou J, Wong BHY, et al. (2016) Middle East respiratory syndrome coronavirus efficiently infects human primary $\mathrm{T}$ lymphocytes and activates the extrinsic and intrinsic apoptotic pathways. J. Infect. Dis. 213: 904:914.

6. Cipolla-Neto J, Amaral F. (2018) Melatonin as a hormone: new physiological and clinical insights. Endocr. Rev. 39: 990-1028.

7. Tricoire H, Locatelli A, Chemineau P, et al. (2002) Melatonin enters the cerebrospinal fluid through the pineal recess. Endocrinology 143: 84-90. 
8. Reiter RJ, Tan DX, Kim SJ, et al., (2014) Delivery of pineal melatonin to the brain and SCN: role of canaliculi, cerebrospinal fluid, tanycytes and Virchow-Robin perivascular spaces. Brain Struct. Funct. 219: 1873-1887.

9. Acuna-Castroviejo D, Escames G, Venegas C, et al. (2014) Extrapineal melatonin: sources, regulation, and potential functions. Cell. Mol. Life Sci. 71: 2997-3025.

10. Poeggeler B, Hardeland R. (1994) Detection and quantification of melatonin in a dinoflagellate, Gonyaulax polyedra: solutions to the problems of methoxyindole destruction in non-vertebrate material. J. Pineal Res. 17: 1-10.

11. Manchester LC, Poeggeler B, Alvares FL, et al. (1995) Melatonin immunoreactivity in the photosynthetic prokaryote Rhodospirillum rubrum: implications for an ancient antioxidant system. Cell. Mol. Biol. Res. 41: 391-395.

12. Zimorski V, Ku C, Martin WF, et al. (2014) Endosymbiotic theory for organelle origins. Curr. Opin. Microbiol. 22: 38-48.

13. Tan DX, Manchester LC, Liu X, et al. (2013) Mitochondria and chloroplasts as the original sites of melatonin synthesis: a hypothesis related to melatonin's primary function in evolution in eukaryotes. J. Pineal Res. 54: 127-138.

14. Venegas C, Garcia JA, Escames G, et al. (2012) Extrapineal melatonin: analysis of its subcellular distribution and daily fluctuations. J. Pineal Res. 52: 217-227.

15. He C, Wang J, Zhang Z, et al. (2016) Mitochondria synthesize melatonin to ameliorate its function and improve mice oocyte's quality under in vitro conditions. Int. J. Mol. Sci. 17: E939.

16. Suofu Y, Jean Alphonse FG, Jia J, et al. (2017) Dual role of mitochondria in producing melatonin and driving GPCR signaling to block cytochrome c release. Proc. Nat. Acad. Sci. USA 114: E7997-E8006.

17. Reiter RJ, Sharma R, Ma Q, et al. (2020) Circadian and non-circadian melatonin: influence on glucose metabolism in cancer cells. J. Curr. Sci. Technol. 10: 85-98.

18. Reiter RJ, Ma Q, Sharma R. (2020) Melatonin in mitochondria: mitigating clear and present dangers. Physiology (Bethesda) 35: 86-95.

19. Reiter RJ, Sharma R, Ma Q, et al. (2020) Melatonin inhibits Warburg-dependent cancer by redirecting glucose oxidation to the mitochondria: a mechanistic hypothesis. Cell. Mol. Life Sci., in press.

20. Yamamoto T, Takano N, Ishiwata K, et al. (2018) Reduced methylation of PFKFB3 in cancer cells shunts glucose towards the pentose phosphate pathway. Nat. Commun. 5: 3480 .

21. Okatani Y, Wakatsuki A, Reiter RJ, et al. (2003) Acutely administered melatonin restores hepatic mitochondrial physiology in old mice. Int. J. Biochem. Cell. Biol. 35: 367-375.

22. Jou MJ, Peng TI, Yu PZ, et al. (2007) Melatonin protects against common deletion of mitochondrial DNA-augmented mitochondria oxidative stress and apoptosis. J. Pineal Res. 43: 389-403.

23. Hevia D, Gonzalez-Menendez P, Quiros-Gonzalez I, et al. (2015) Melatonin uptake through glucose transporters: a new target for melatonin inhibition of cancer. J. Pineal Res. 58: 234250.

24. Huo X, Wang C, Yu Z, et al. (2017) Human transporters, PEPT1/2, facilitate melatonin transportation into mitochondria of cancer cells: an implication of the therapeutic potential. $J$. Pineal Res. 62: 12390.

25. Acuna-Castroviejo D, Noguiera-Navarro MT, Reiter RJ, et al. (2018) Melatonin actions in the heart; more than a hormone. Melatonin Res. 1: 21-26. 
26. Reiter RJ, Rosales-Corral S, Tan DX, et al. (2017) Melatonin as a mitochondria-targeted antioxidant: one of evolution's best ideas. Cell. Mol. Life Sci. 74: 3863-3881.

27. Reiter RJ, Mayo JC, Tan DX, et al. (2016) Melatonin as an antioxidant: under promises but over delivers. J. Pineal Res. 61: 253-278.

28. Blask DE, Dauchy RT, Dauchy EM, et al. (2014) Light exposure at night disrupts host/cancer circadian regulatory dynamics: impact on the Warburg effect, lipid signaling and tumor growth prevention. PLoS One 9: e102776.

29. Tan DX, Reiter RJ. (2019) Mitochondria: the birth place, battle ground and the site of melatonin metabolism in cells. Melatonin Res. 2: 44-66.

30. Reiter RJ, Rosales-Corral S. (2019) Melatonin reprograms glucose metabolism in cancer cell mitochondria. Series Endocrinol. Diabetes Metab. 1: 52-61.

31. Reiter RJ, Sharma R, Ma Q, et al. (2019) Inhibition of pyruvate dehydrogenase kinase: a proposed mechanism by which melatonin causes cancer cells to overcome cytosolic glycolysis, reduce tumor biomass and reverse insensitivity to chemotherapy. Melatonin Res. 2: 105-119.

32. Courtnay R, Ngo DC, Malik N, et al. (2015) Cancer metabolism and the Warburg effect: the role of HIF-1 and PI3K. Mol. Biol. Rep. 42: 841-851.

33. Jardim-Perassi BV, Alexandre PA, Sonehara NM, et al. (2019) RNA-Seq transcriptome analysis shows anti-tumor actions of melatonin in a breast cancer xenograft model. Sci. Rep. 9: 966.

34. Ferreira LC, Orso F, Dettori D, et al. (2020) The role of melatonin on miRNAs modulation in triple-negative breast cancer cells. PLoS One 15: e0228062.

35. Zhao D, Yu Y, Shen Y, et al. (2019) Melatonin synthesis and function: evolutionary history in animals and plants. Front. Endocrinol. 10: 249.

36. Klein DC (2007) Arylalkylamine N-acetyltransferase: the timenzyme. J. Biol. Chem. 282: 4233-4237.

37. Anderson G. (2019) Daytime orexin and night-time melatonin regulation of mitochondria melatonin: roles in circadian oscillations systemically and centrally in breast cancer symptomatology. Melatonin Res. 2: 1-8.

38. Anderson G, Maes M, Markus RP, et al. (2015) Ebola virus: melatonin as a readily available treatment option. J. Med. Virol. 87: 537-543.

39. Anderson G, Reiter RJ. (2019) Glioblastoma: role of mitochondria Nacetylserotonin/melatonin ratio in mediating effects of miR-451 and aryl hydrocarbon receptor and in coordinating wider biochemical changes. Int. J. Tryptophan Res. 12: 1-9.

40. Arabi YM, Fowler R, Hayden FG. (2020) Critical care management of adults with community-acquired severe respiratory viral infection. Intensive Care Med. 46: 315-328.

41. Grant WB, Lahore H, McDonnell SL, et al. (2020) Evidence that vitamin D Supplementation could reduce risk of influenza and COVID-19 infection and deaths. Nutrients 12: E988.

42. Marik PE, Kory P, Varon J. (2020) Does vitamin D status impact mortality from SARSCoV-2 infection? Med. Drug Discov. 29: 100041.

43. Martin-Gimenez VM, Inserra F, Tajer CE, et al. (2020) Lungs as target of COVID-19 infection: protective common molecular mechanisms of vitamin D and melatonin as a potential synergistic treatment. Life Sci. 254: 117808.

44. Wu C, Chen X, Cai Y, et al. (2020) Risk factors associated with acute respiratory distress syndrome and death in patients with coronavirus disease 2019 pneumonia in Wuhan, China. JAMA Intern. Med. 13: e200994. 
45. Mehta P, McAuley DF, Brown M, et al. (2020) COVID-19: Consider cytokine storm syndromes and immunosuppression. Lancet 395: 1033-1934.

46. Martins E Jr, Ferreira AL, Skorupa AL, et al. (2004) Tryptophan consumption and indoleamines production in peritoneal cavity macrophages. J. Leukoc. Biol. 75: 1116-1121.

47. Muxel SM, Pires-Lapa MA, Monteiro AW, et al. (2012) NF-kB drives the synthesis of melatonin in RAW 264.7macrophages by inducing the transcription of the arylalkylamine Nacetyltransferase (AA-NAT) gene. PLoS One 7: E52010.

48. Muxel SM, Laranjeira-Silva MF, Carvalho-Sousa CE, et al. (2016) The RelA/cRel nuclear factor-kB (NF-kB) dimer, crucial for inflammation resolution, mediates the transcription of the key enzyme in melatonin synthesis in RAW 264:7 macrophages. J. Pineal Res. 60: 394-404.

49. Carvalho-Sousa CE, Pereira EP, Kinker GS, et al. (2020) Immune-pineal axis protects rat lungs exposed to polluted air. J. Pineal Res. 68: e12636.

50. Gordon S. (1995) The macrophage. Bioessays 17: 977-986.

51. Osborn O, Olefsky JM (2012) The cellular and signaling networks linking the immune system and metabolism in disease. Nat. Med. 18: 363-374.

52. Gordon S, Martinez FO. (2010) Alternative activation of macrophages: mechanism and functions. Immunity 32: 593-604.

53. Rodriguez-Prados JC, Traves PG, Cuenca J, et al. (2010) Substrate fate in activated macrophages: a comparison between innate, classic, and alternative activation. J. Immunol. 185: 605-614.

54. Hardeland R. (2018) Melatonin and information-story of a double-edged blade. J. Pineal Res. 65: e12525.

55. Perez-Gonzalez A, Castaneda-Arriaga R, Alvarez-Idaboy JR, et al. (2019) Melatonin and its metabolites as chemical agents capable of directly repairing oxidized DNA. J. Pineal Res. 66: e12539.

56. Tan DX, Hardeland R. (2020) Potential utility of melatonin in deadly infectious diseases related to the overreaction of innate immune system response and destructive inflammation: focus on COVID-19. Melatonin Res. 3: 120-143.

57. Reiter RJ, Sharma R, Ma Q, et al. (2020) Melatonin inhibits COVID-19-induced cytokine storm by reversing aerobic glycolysis in immune cells: a mechanistic analysis. Med. Drug. Discov., in press.

58. Behrens EM, Koretzky GA (2017) Review: Cytokine storm syndrome: looking forward toward the precision medicine era. Arthritis Rheumatol. 69: 1135-1143.

59. Tugal D, Liao X, Jain MK. (2013) Transcriptional control of macrophage polarization. Arterioscler. Thromb. Vasc. Biol. 33: 1135-1144.

60. Essandoh K, Li Y, Huo J, et al. (2016) MiRNA-mediated macrophage polarization and its potential role in the regulation of inflammatory response. Shock 46: 122-131.

61. Tobias PS, Soldau K, Ulevitch RJ. (1989) Identification of a lipid A binding site in the acute phase reactant lipopolysaccharide binding protein. J. Biol. Chem. 264: 10867-10871.

62. Mauriz JL, Collado PS, Veneroso C, et al. (2013) A review of the molecular aspects of melatonin's anti-inflammatory actions: recent insights and new perspectives. J. Pineal Res. 54: 1-14.

63. Liu Z, Gan L, Xu Y, et al. (2017) Melatonin alleviates inflammasome-induced pyroptosis through inhibiting NF-kB/GSDMD signal in mice adipose tissue. J. Pineal Res. 63: 12414. 
64. Yi WJ, Kim TS. (2017) Melatonin protects mice against stress-induced inflammation through enhancement of M2 macrophage polarization. Int. Immunopharmacol. 48: 146-158.

65. Amici SA, Dong J, Guerau-de-Arellano M. (2017) Molecular mechanisms modulating the phenotype of macrophages and microglia. Front. Immunol. 8: 1520.

66. Carloni S, Favrais G, Saliba E, et al. (2016) Melatonin modulates neonatal brain inflammation through endoplasmic reticulum stress, autophagy and miR-34a/silent information regulator 1 pathway. J. Pineal Res. 61: 370-380.

67. Ding S, Lin N, Sheng X, et al. (2019) Melatonin stabilizes rupture-prone vulnerable plaques via regulating macrophage polarization in a nuclear circadian receptor $\mathrm{ROR} \alpha$-dependent manner. J. Pineal Res. 67: e12581.

68. Xia Y, Chen S, Zeng S, et al. (2019) Melatonin in macrophage biology: current understanding and future perspectives. J. Pineal Res. 66: e12547.

69. Liu ZJ, Ran YY, Qie SY, et al. (2019) Melatonin protects against ischemic stroke by modulating microglia/macrophage polarization toward anti-inflammatory phenotype through STAT3 pathway. CNS Neurosci. Ther. 25: 1353-1362.

70. Thomas MC, Pickering RJ, Tsorotes D, et al. (2010) Genetic Ace2 deficiency accentuates vascular inflammation and atherosclerosis in the ApoE knockout mouse. Circ. Res. 107: 888-897.

71. McGonagle D, Sharif K, O'Regan A, et al. (2020) The role of cytokines including interleukin-6 in COVID-19 induced pneumonia and macrophage activation syndrome-like disease. Autoimmun. Rev. 2020: 102537.

72. He L, Ding Y, Zhang Q, et al. (2006) Expression of elevated levels of pro-inflammatory cytokines in SARS-CoV-infected ACE2+ cells in SARS patients: relation to acute lung injury and pathogenesis of SARS. J. Pathol. 210: 288-297.

73. Jia H (2016) Pulmonary angiotensin-converting enzyme 2 (ACE2) and inflammatory lung disease. Shock 46: 239-248.

74. Imai Y, Kuba K, Rao S, et al. (2005) Angiotensin-converting enzyme 2 protects from severe acute lung failure. Nature 436: 112-116.

75. Zhang R, Yu Y, Ni L, et al. (2020) COVID-19: melatonin as a potential adjuvant treatment. Life Sci. 250: 117583.

76. Anderson G, Reiter RJ. (2020) Melatonin: roles in influenza, COVID-19 and other viral infections. Rev. Med. Viral. 21: e2109.

77. Shneider A, Kudriavtsev A, Vakhrusheva A. (2020) Can melatonin reduce the severity of COVID-19 pandemic? Int. Rev. Immunol. 29: 1-10.

78. Reiter RJ, Abreu-Gonzalez P, Marik PE, et al. (2020) Therapeutic algorithm for the use of melatonin in patients with COVID-19. Front. Med. 7: 226.

79. Tain YL, Huang LT, Hsu CN, et al. (2014) Melatonin therapy prevents programmed hypertension and nitric oxide deficiency in offspring exposed to maternal calorie restriction. Oxid. Med. Cell. Longev. 2014: 283180.

80. Zou Z, Yun Y, Shu Y, et al. (2014) Angiotensin-converting enzyme 2 protects from lethal avian influenza A H5N1 infections. Nat. Commun. 5: 3594.

81. Gu H, Xie Z, Li T, et al. (2016) Angiotensin-converting enzyme 2 inhibits lung injury induced by respiratory syncytial virus. Sci. Rep. 6: 19840.

82. Abedi F, Hayes AW, Reiter R, et al. (2020) Acute lung injury: the therapeutic role of Rho kinase inhibitors. Pharmacol. Res. 155: 104736. 
83. Borin TF, Arbab AS, Gelaleti GB, et al. (2016) Melatonin decreases breast cancer metastasis by modulating Rho-associated kinase protein-1 expression. J. Pineal Res. 60: 3-15.

84. Liu Z, Zou D, Yang X, et al. (2017) Melatonin inhibits colon cancer RKO cell migration by downregulating Rho-associated protein kinase expression via the p38/MAPK signaling pathway. Mol. Med. Rep. 16: 9383-9392.

85. Su SC, Hsieh MJ, Yang WE, et al. (2017) Cancer metastasis: mechanisms of inhibition by melatonin. J. Pineal Res. 62: 12370.

86. Boga JA, Coto-Montes A, Rosales-Corral SA et al. (2012) Beneficial actions of melatonin in the management of viral infections: a new use for this "molecular handyman"? Rev. Med. Virol. 22: 323-338.

87. Lu R, Liu Z, Shao Y, et al. (2019) Melatonin is responsible for rice resistance to rice stripe virus infection through a nitric oxide-dependent pathway. Virol. J. 16: 141.

88. Zhou Y, Hou Y, Shen J, et al. (2020) Network-based drug repurposing for novel coronavirus 2019-nCoV/SARS-CoV-2. Cell Discov. 6: 14.

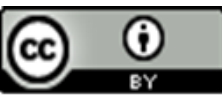

This work is licensed under a Creative Commons Attribution 4.0 International License

Please cite this paper as:

Reiter, R.J., Sharma, R., Ma, Q., Liu, C., Manucha, W., Abreu-Gonzalez, P. and DominguezRodriguez, A. 2020. Plasticity of glucose metabolism in activated immune cells: advantages for melatonin inhibition of COVID-19 disease. Melatonin Research. 3, 3 (Jun. 2020), 362-379. DOI:https://doi.org/https://doi.org/10.32794/mr11250068. 